\title{
Reliability Evaluation of High Speed Train Bogie System Based on Stochastic Network Flow Model
}

\author{
Vartika Sharma \\ School of Information and Communication Technology, Gautam Buddha University, Greater Noida, Uttar Pradesh, \\ 201-308, India \\ Email: vartika.sharma0510@gmail.com
}

\section{Rajesh Mishra}

School of Information and Communication Technology, Gautam Buddha University, Greater Noida, Uttar Pradesh, 201-308, India

Received: 01 May 2020; Accepted: 13 May 2020; Published: 08 October 2020

\begin{abstract}
Bogie is an emblematic complex mechanical as well as electronic part of the current high speed rail system. A small failure of components may lead to a loss of production, casualties and damage of the system. Therefore, security and reliability analysis of bogie system is a predominant task. This paper proposes a stochastic network flow model of bogie system based on the forces applied on the components of the bogie, simultaneously considering the deterioration level and functional correlation of components. At first, a detailed description of structure and functions of the CRH3 bogie is introduced. Then a stochastic network flow model is constructed by analysing the direction of various forces applied on bogie. In the proposed model, edges represent the bogie components and vertices are the transmission channels. The flow over each edge is analysed by the forces it withstands. Finally, a combination method using minimal cut sets is proposed to evaluate the reliability of high speed train bogie system. This paper provides a supportive guidance and practical approach to bogie system designers for efficient operation and maintenance of the bogie.
\end{abstract}

Index Terms: High Speed Train, Bogie, Reliability Evaluation, Minimal Cut sets, Stochastic Flow Networks.

\section{Introduction}

Railway is a prominent transportation system for importing and exporting a huge volume of goods and increasing number of passengers from years. With a great advancement in structure and functioning of railway system, it is essential to provide the safety and reliability as well. The term safety and reliability is directly influenced by the bogie system of today's high speed rail vehicle. Bogie is an emblematic complex mechanical as well as electronic part of the rail vehicle which is quickly prone to failure. A minor failure in bogie components may direct to causality, loss of investment and system damage. Therefore, the reliability analysis of bogie system is a predominant task and has emerged as a most progressive research area in railway field.

There is very limited work available in literature to evaluate the reliability of bogie system. Some of the conventional methods are reliability block diagrams (RBD) [1], Markov analysis [2], Petri nets [3], fault tree analysis [4] and failure mode and effect analysis and derivatives (FMEA) [5]. But these methods are restricted for bogie system reliability evaluation since the bogie is a complex electromechanical system and these are preferable for single component reliability evaluation of a bogie. In [6], the author evaluated the reliability of bogie system based on copula theory. But they have considered the network as a binary system, which is not realistic in real time application. Seo. et.al. [5] used fault tree analysis (FTA) and failure mode and effect analysis (FMEA) method to calculate the reliability of metro traction power system based on minimal cut sets. Further, in [7], the author improved the connectivity reliability of bogie using two states i.e. success and failure, but again it was a binary model. After words, in [8], the author proposed a novel method for bogie system reliability evaluation using complex network theory. They have described the three types of connections among bogie components as electrical, mechanical and information connection. In order to evaluate the system reliability, they have introduced the concept on network agglomeration. Most of the papers existing in literature considers the bogie system as binary but in practical scenario, all the components cannot be fully functional over time, their capacity degrades on various levels to complete failure. Hence, in this paper, the authors have presented the bogie system as a stochastic network 
flow model working on various capacity levels to fulfil the fixed given demand from source to destination. The proposed network model has a uniqueness from general trend as the arcs denote components of bogie system and vertices represent the force transmission among components.

Hypothetically, two terminal reliability evaluation of a stochastic flow networks is an NP-hard problem [9]. A lot of algorithms are available in literature including approximation algorithm [10] and exact evaluation algorithms [11, 12] to provide the solution for stochastic network reliability problems. Most of the approaches are based on d-minimal path sets [11], [13-15] and d-minimal cut sets [16-18]. Then finally these generated d-minimal path sets (cutsets) are used to evaluate exact reliability of a network using any of the method such as Inclusion-Exclusion, Sum of Disjoint Product (SDP) etc. In a stochastic flow network even though the network is connected, it is not compulsory that the network is able to transfer the desired amount of flow unless the capacity states of intermediate nodes is capable of transferring the desired flow. Therefore, minimal cutsets are used to check the blocking capacity of the network for demand d. In this paper, a simple combination based reliability evaluation approach is proposed using minimal cutsets since minimal cutsets are the minimal set of links upon removal will disconnect the source and terminal node.

The upcoming sections are arranged in the following order. Section 2 presents the concise description of the bogie system and the stochastic flow network. Network modelling and proposed methodology is detailed in Section 3. Results and discussion is provided in Section 4 followed by the conclusion in Section 5.

\section{Related Work}

This section presents the basic knowledge of various bogie components and their functions along with the brief introduction of stochastic flow networks. The current relevance and motivation of performing this research is also showcased in this section.

\subsection{Bogie System}

Bogie is a part of rail vehicle which is normally ignored by the passengers but even though this ambiguity, it is extremely important for the smooth and safe functioning of railway operations. The basic functions of a bogie are:

- It supports rail car body to run rigidly on both curved and straight track.

- It provides a comfortable drive by accumulating the irregularities and vibrations generated on tracks.

- Bogie system also supports in reducing the centrifugal force while train runs on a curved track with High speed.

There are various classifications available in literature for bogie structure [20]. For example, bogies can be classified based on number of axles such as single axle, double axle, triple axle etc. Based on suspension, there is another classification i.e. non-articulated and articulated bogies. Articulated bogies support two rail car while non-articulated bogies supports only one rail car. Most of the high speed trains such as Spanish Talgo, French TGV are enabled with articulated bogies. Again, based on the structure of the suspension gear, bogies can be divided into two types as swing hanger bogie and small lateral stiffness bolster spring bogie. Also, there is another classification i.e. bolster and bolster less bogies. The components of a bogie system varies according to the type and functionality of bogie, but the fundamental components of a bogie are wheel set, bogie frame, primary suspension, secondary suspension, bump-stop, yaw damper, vertical damper, lateral damper, brake system and so on as shown in Fig. 1.

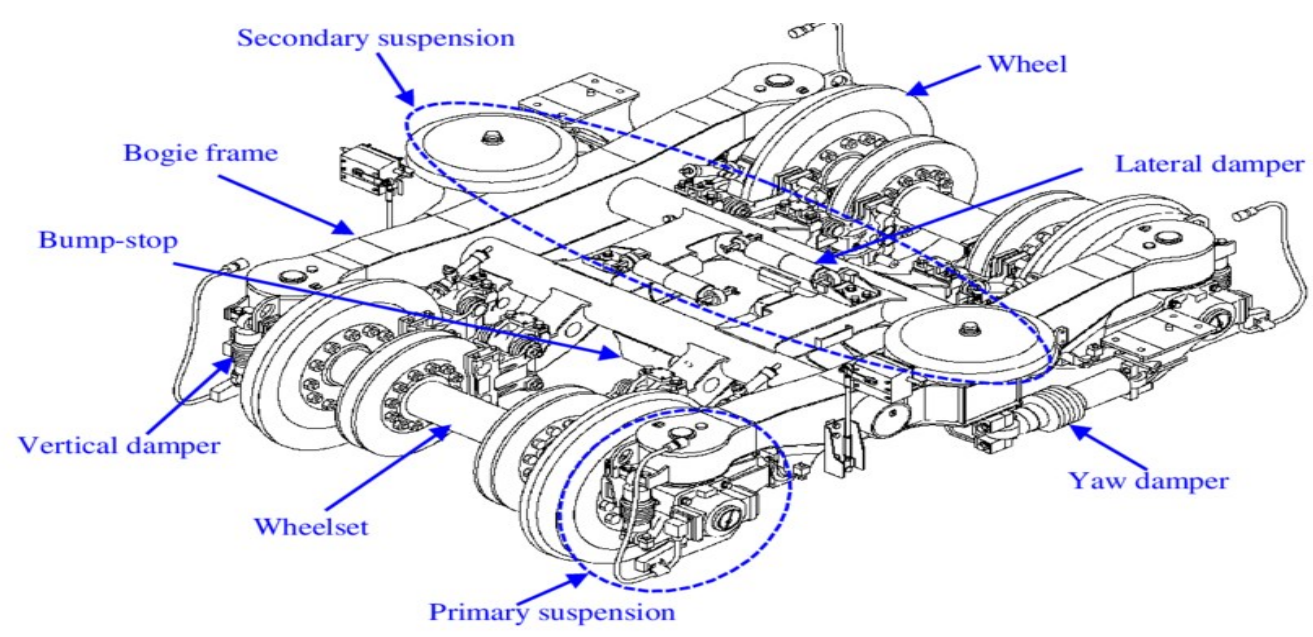

Figure 1: Components of a Chinese High Speed Railway Bogie [19]

The bogie frame provides shelter to various bogie parts and supports the load. Wheels are connected with the axles 
through bearing and are susceptible to mass imbalance. The most critical part of a bogie is suspension gear which includes bolster spring, yaw-damper, lateral damper and traction transfer device. It is the subsystem which is responsible for active conduct of bogie, comfortable drive and body support. It accumulates the shocks generated between wheel and bogie frame while running on curved tracks with high speed. There are basically two types of suspension gear i.e. primary suspension also known as coil springs and secondary suspension, known as rubber airbags. (Detailed description is provided in [20]).

This paper focuses on the transmission of force among above mentioned mechanical components. The direction and quantity of force applied on these components works as a flow. Since they are mechanical components, they cannot be fully functional over time, so their working capability is considered as stochastic. Hence, this paper visualize the force transmission among bogie components as a stochastic flow network and evaluates the reliability among source-destination pair with fixed demand.

\subsection{Stochastic Flow Networks}

Stochastic flow network can be defined as a directed graph $G(V, E, C)$, where $V$ is the set of vertices with $V=\left\{v_{i} \mid 1\right.$ $\leq i \leq n\}$ and $s, t \in V$. $E$ is the set of edges with $E=\left\{e_{i} \mid 1 \leq i \leq n\right\}$ and $C$ is the set of $a_{i}$ 's capacity states with $C=\left\{c_{i} \mid 1 \leq i\right.$ $\leq n\}$ as shown in Fig. 2.

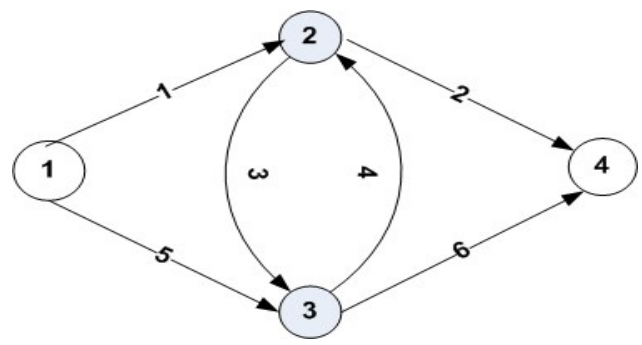

Figure 2: A 4N6L stochastic flow network

In a stochastic network, each and every link has multiple, independent capacity states ranging from o to $\mathrm{n}$ along with their respective probabilities as shown in Table 1. For example, link a1 has four capacity states $0,1,2$ and 3 with their respective probability distribution $0.05,0.10,0.15$ and 0.70 . If link a1 is working on capacity state 0 , then it is considered as failed and on other hand, if it is working on 3, then identifies as fully operational. Terminal pair reliability of a stochastic network can be calculated as the probability that the maximum flow from source to destination node should not be less than the given demand $\mathrm{d}$. In other words, the probability that the network is capable of transmitting the demand d from source to destination node.

Table 1: Capacity state probability distribution of Fig. 2

\begin{tabular}{c|r|r|r|r|r|r|r|c}
\hline Link & \multicolumn{5}{|c|}{ Capacity } & \multicolumn{4}{c}{ Probability } \\
\hline $\mathrm{a} 1$ & 0 & 1 & 2 & 3 & 0.05 & 0.10 & 0.15 & 0.70 \\
\hline $\mathrm{a} 2$ & 0 & 1 & 2 & - & 0.10 & 0.15 & 0.75 & - \\
\hline $\mathrm{a} 3$ & 0 & 1 & - & - & 0.05 & 0.95 & - & - \\
\hline $\mathrm{a} 4$ & 0 & 1 & - & - & 0.05 & 0.95 & - & - \\
\hline $\mathrm{a} 5$ & 0 & 1 & - & - & 0.10 & 0.90 & - & - \\
\hline $\mathrm{a} 6$ & 0 & 1 & 2 & - & 0.05 & 0.10 & 0.85 & - \\
\hline
\end{tabular}

\subsection{Motivation}

Reliability evaluation of real world networks such as transportation networks [17], power distribution networks [21], social networks [22], computer communication networks [23] etc. is a prominent task to analyse the quality of service. All above mentioned networks are regarded as stochastic flow networks where components can work on various degradation levels to complete failure. This fundamental property is applicable on mechanical systems as well. They also work on multiple working states, hence, in this paper we have taken the advantage of stochastic property of mechanical systems and applied our reliability evaluation approach on high speed rail bogie system. Up to best of our knowledge, bogie system reliability evaluation approach considering stochastic capacity of mechanical components is hardly available in literature. 


\section{Proposed Methodology}

This section provides the detailed description of modeled bogie network with essential assumptions. Proposed methodology of bogie system reliability evaluation is explained with illustrative model in the following section.

\subsection{Network Modelling}

This paper evaluates the reliability of a high speed china railway (CRH3) bogie and considers the mechanical operation only. Apart from supporting the vehicle body, another functions of bogie are traction transfer, absorbing vibrations on curved tracks, control of braking force etc. [24]. Hence, after analysing these functions, the direction and amount of force applied on various bogie components represent the flow in bogie system. According to [25], forces transferred by bogie components can be classified into three types.

- The vertical force, which is applied by the vehicle body on rubber airbags to bogie frame and bogie frame creates the pressure on coil springs. From there it goes to axle box and then comes on wheels which generates vertical vibrations.

- The longitudinal force, which is developed in the opposite direction of vertical force.

- The lateral force, which is provoked by the centrifugal force and the force generated when the train runs on a curved track with high speed.

The flow of these forces [25] is used to visualize the network structure of the bogie system as shown in Fig. 3 and Fig. 4.

In Fig. 4, the edges represents the mechanical components of the bogie and vertices denote the force transmission path among the components. Dotted line denotes the rail vehicle which supports the wheels in vertical force direction. Since we are considering the deterioration level of components i.e. in practical, it is observed that capacity of bogie parts reduces after operating long time, Table 2 denotes the degraded capacity states of various bogie components along with their probability distribution. For example, as shown in Table 2 brake system is classified into four states and the working probability of each state is $0.05,0.10,0.25$ and 0.60 respectively.

In order to evaluate the stochastic network reliability of bogie system, the authors have considered the following assumptions.

- Nodes are statically independent and perfectly reliable, i.e. connections among bogie parts are always working.

- The flow in the network satisfies the flow conservation law [26].

- Since force is applicable in a specific direction, network is considered as a directed network.

- Capacity of each component is a non-negative integer value followed by a probability distribution.

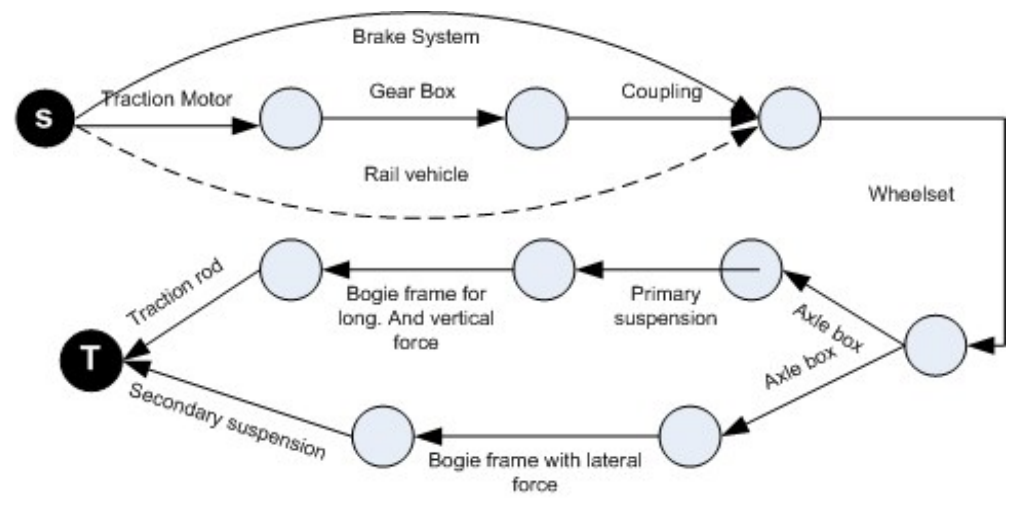

Figure 3: Force broadcasting in bogie system

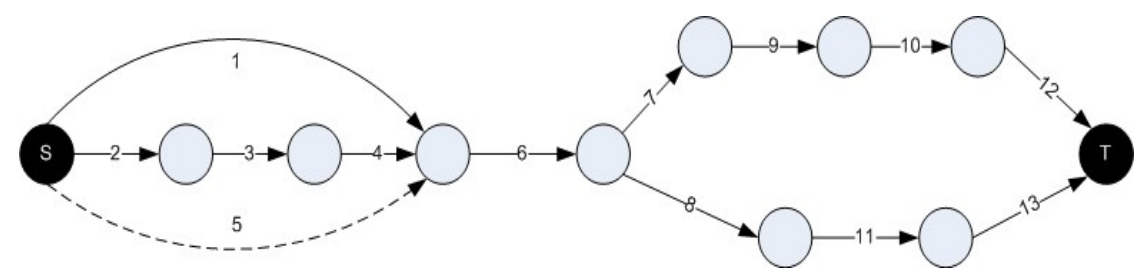

Figure 4: Network modelling of bogie system [25] 
Table 2. Capacity Probability Distribution Data of bogie components

\begin{tabular}{ccccccccc}
\hline \multicolumn{3}{l}{ Links } & \multicolumn{3}{c}{ Capacity } & & \multicolumn{5}{l}{ Probability Distribution } \\
\hline 1 & 0 & 4 & 7 & 10 & 0.05 & 0.10 & 0.25 & 0.60 \\
2 & 0 & 5 & 10 & - & 0.10 & 0.30 & 0.60 & - \\
3 & 0 & 3 & 7 & 10 & 0.05 & 0.10 & 0.20 & 0.65 \\
4 & 0 & 10 & - & - & 0.10 & 0.90 & - & - \\
5 & 0 & 10 & - & - & 0 & 1 & - & - \\
6 & 0 & 18 & 24 & 30 & 0.05 & 0.25 & 0.50 & 0.20 \\
7 & 0 & 10 & 16 & 20 & 0.10 & 0.20 & 0.25 & 0.45 \\
8 & 0 & 5 & 8 & 10 & 0.10 & 0.20 & 0.25 & 0.45 \\
9 & 0 & 12 & 17 & 20 & 0.05 & 0.05 & 0.15 & 0.75 \\
10 & 0 & 16 & 20 & - & 0.10 & 0.20 & 0.70 & - \\
11 & 0 & 8 & 10 & - & 0.10 & 0.20 & 0.70 & - \\
12 & 0 & 9 & 15 & 20 & 0.05 & 0.10 & 0.15 & 0.70 \\
13 & 0 & 4 & 7 & 10 & 0.05 & 0.15 & 0.35 & 0.45 \\
\hline
\end{tabular}

\subsection{Proposed Approach}

The proposed approach is a three step process. The first step obtains the minimal cutsets of the bogie network shown in Fig. 4. Next step reduces the number of minimal cutsets by eliminating the redundant ones in order to reduce the computation. Finally, the last step evaluates the reliability of the network by scanning and calculating each minimal cutsets reliability using combinatorial method. The detailed description of the proposed approach is as follows.

1) Obtaining Minimal Cutsets: The first step starts with the generation of minimal cutsets using the approach proposed by [27]. In this approach, node cutsets are identified first. Node cutsets are the set of node which are directly connected to each other and after removal of these nodes, remaining stochastic network will be divided into two parts i.e. one part will include the node set and another one will contain the remaining network. Since the number of nodes are less as compared to number of links in a practical network [27], hence, it is efficient to calculate node cutsets as compared to finding link cutsets. Now from the generated node sets only specific node sets will produce the minimal cutsets. These node stes are known as node cutsets. Each node set is associated with two specific notations, nHigh and nMax. nHigh of a node set is the highest node number in that node set and nMax is the highest node number connected to the node of that node set.

Table 3: Minimal cutsets of the network Fig. 4

\begin{tabular}{rccc}
\hline Cut set No. & Minimal Cut Set & Cut set No. & Minimal Cut Set \\
\hline 1 & $\{6\}$ & 9 & $\{8,10\}$ \\
2 & $\{1,2,5\}$ & 10 & $\{8,12\}$ \\
3 & $\{1,3,5\}$ & 11 & $\{9,11\}$ \\
4 & $\{1,4,5\}$ & 12 & $\{9,13\}$ \\
5 & $\{7,8\}$ & 13 & $\{10,11\}$ \\
6 & $\{7,11\}$ & 14 & $\{10,13\}$ \\
7 & $\{7,13\}$ & 15 & $\{11,12\}$ \\
8 & $\{8,9\}$ & 16 & $\{12,13\}$ \\
\hline
\end{tabular}

Example 1. Consider the stochastic flow network of Fig. 2. For the node set $\{2\}$, nHigh is 2 and nMax is 3 . For the node set $\{3\}$, nHigh is 3 and nMax is 3 . Hence, the node set with cardinality 1 are $\{1\},\{2\}$ and $\{3\}$.

For higher order generation node sets, include the additional node numbers from nHigh +1 to nMax and continue the process up to (n-1) generation.

Example 2. Consider the network in Fig. 2. For the first order node set $\{1\}$, next order possible node sets are $\{1,2\}$, $\{1,3\}$. For $\{1,2\}$, next order node set are $\{1,2,3\}$.

Next, the obtained node cut sets are converted into link cut sets by performing the set-theoretic X-ORing of link sets. Hence, by applying the above mentioned approach on Fig.4, obtained minimal cutsets for bogie system are shown in Table 3. 
2) Obtain the Ir-redundant Minimal Cutsets: Since the combinatorial method is used for reliability evaluation, we will scan each and every link present in the cut sets and make the combination with the link newly introduced. Once all the links present in the network are included, new combinations cannot be generated by the remaining minimal cuts. Hence, the remaining cut sets are concluded as redundant cut sets.

Example 3. Consider the Cut set No. 11, \{9, 11\} from Table 3. There is no link present in this cut set which is not considered before, therefore, no new combination can be generated from this cut set. Hence, $\{9,11\}$ is a redundant cut set.

The redundancy removal of minimal cut sets will help in rapid reliability evaluation of bogie system by reducing the runtime. Table 4 gives the list of obtained irredundant minimal cut sets.

Table 4: Irredundant minimal cut sets of the network Fig. 4

\begin{tabular}{cccc}
\hline Cut set No. & Minimal Cut sets & Cut set No. & Minimal Cut sets \\
\hline 1 & $\{6\}$ & 6 & $\{7,11\}$ \\
2 & $\{1,2,5\}$ & 7 & $\{7,13\}$ \\
3 & $\{1,3,5\}$ & 8 & $\{8,9\}$ \\
4 & $\{1,4,5\}$ & 9 & $\{8,10\}$ \\
5 & $\{7,8\}$ & & 10 \\
\hline
\end{tabular}

3) Reliability Calculation: Finally, the last step deals with the reliability evaluation of the bogie system using the obtained minimal cut sets. Each cut will be scanned one by one in order to evaluate the unreliability of that particular cut. Finally, unreliability of all the cuts will be sum up to evaluate the unreliability of whole system.

The process will start with the formation of all possible capacity combination of the links present in the first cut. Add the capacities of each combination and evaluate the probability value for each combination. Now, select the combinations whose total capacity is less than the given demand $\mathrm{d}$. Add the probabilities of the selected combinations. The resultant probability will denote the unreliability of that particular cut set. Store the remaining combinations in an array residual comb. These combinations are the successful links which are able to transfer the demand $d$.

Example 4. Consider the 5th cut set $\{7,8\}$ from Table 4. Suppose the demand dto be passed across the network is 30 units. Possible combinations of this cut set are $[(0,0),(0,5),(0,8),(0,10),(10,0),(10,5),(10,8),(10,10)$, $(16,0),(16,5),(16,8),(16,10),(20,0),(20,5),(20,8)$, and $(20,10)]$. Probability of each combinations are [0.01, 0.02, $0.025,0.045 \ldots . . .0 .09,0.1125]$.

Now the selected combinations with total capacity less than demand 30 units are $[(0,0),(0,5),(0,8),(0,10),(10,0),(10,5),(10,8),(10,10),(16,0),(16,5),(16,8),(16,10),(20,0),(20,5),(20,8)]$. Combination (20,10) is containing capacity equal to demand, so this combination is not included and is stored in residual comb. Summation of the probability of all the selected combination will give the unreliability of the 5th cut set $\{7,8\}$. This is the probability of failure of the network due to the failure of $\{7,8\}$ cut set.

Similarly, next cut set will be scanned and new link present in this cut set will be combined with the residual comb of the previous cut. The process repeats and continues until the unreliability calculation of all the cut sets. Finally, unreliability value is converted into the reliability expression using the formula, Network Reliability $=1$ - Unreliability, for the given demand $d$.

Table 5: Bogie system reliability under various demand $d$

\begin{tabular}{cccc}
\hline Demand & Reliability (R) & Demand & Reliability ( R ) \\
\hline 20 & 0.27929399 & 22 & 0.15743826 \\
24 & 0.083547438 & 26 & 0.00962559 \\
28 & 0.001974746 & 30 & 0.00098737309 \\
\hline
\end{tabular}

The reliability of bogie system of Fig. 4 for various demands is shown in Table 5 where $\mathrm{R}$ is the reliability of the bogie running perfectly under the given demand $d$.

\section{Results and Discussion}

The proposed approach is implemented in java (jdk1:8:0:231) using eclipse integrated development environment (IDE) on personal computer with Intel(R) Core (TM) $2.50 \mathrm{GHz}$ CPU. The results are shown in Table 5 which represents the probability that bogie is working in a perfect condition under the certain specific environment. For example, if the demand $d=20, R=0.27929399$, which means if we consider the conditions given in Table 2 and the minimum applicable force from source to destination is 20 units, then the probability of bogie to be in operating is 0.27929399 . The results are verified with [25] to prove the validity of the proposed approach. 


\section{Conclusion}

This paper presents a stochastic network reliability evaluation approach for high speed train bogie system considering the various deterioration capacity levels of mechanical parts of the bogie system. The force applied on various parts of the bogie is analysed and a network model is prepared based on the direction and quantity of force. Since the operational capacity of bogie components varies from fully functional to degrade to completely fail over time, this paper accounts the components state as stochastic network arcs. Further, a minimal cut sets based combination method is proposed to evaluate the reliability of bogie system without any forehead requirement, hence, the method will emerge as an excellent tool for analysing the reliability as well as operation and maintenance of rail vehicle bogie system.

Since there is very limited work available in literature to evaluate the bogie system reliability considering mechanical parts, it is a vast area to work on the real applications of high speed train bogie system to enhance the more practical approaches.

\section{References}

[1] W.-C. Hsu and Y.-T. Chiu, “An application of reliability analysis on the signaling system of the kaohsiung metro,” in 2012 International Symposium on Computer, Consumer and Control. IEEE, 2012, pp. 564-567.

[2] L. R. Doyon, "Markov chains in reliability analysis by computer." 1976.

[3] C. Lijie, T. Tao, Z. Xianqiong, and E. Schnieder, "Verification of the safety communication protocol in train control system using colored petri net,” Reliability engineering \& system safety, vol. 100, pp. 8-18, 2012.

[4] S. Chen, T. Ho, and B. Mao, "Reliability evaluations of railway power supplies by fault-tree analysis," IET Electric Power Applications, vol. 1 , no. 2, pp. 161-172, 2007.

[5] S. Seo, C. S. Park, S. Choi, Y. Han, and K. Kim, "Reliability management and assessment for the electric traction system on the korea highspeed train,” Proceedings of the Institution of Mechanical Engineers, Part F: Journal of Rail and Rapid Transit, vol. 224, no. 3, pp. 179-188, 2010.

[6] X.-Y. Li, Y. Liu, C.-J. Chen, and T. Jiang, "A copula-based reliability modeling for nonrepairable multi-state k-out-of-n systems with dependent components,” Proceedings of the Institution of Mechanical Engineers, Part O: Journal of Risk and Reliability, vol. 230, no. 2, pp. 133-146, 2016.

[7] L. Li, L. Jia, Y. Wang, and J. Li, "Reliability evaluation for complex system based on connectivity reliability of network model," in 2015 International Conference on Logistics, Informatics and Service Sciences (LISS). IEEE, 2015, pp. 1-5.

[8] S. Lin, L. Jia, Y. Wang, Y. Qin, and M. Li, "Reliability study of bogie system of high-speed train based on complex networks theory," in Proceedings of the 2015 International Conference on Electrical and Information Technologies for Rail Transportation. Springer, 2016, pp. 117-124.

[9] M. O. Ball, T. Magnanti, C. L. Monma, and G. L. Nemhauser, Handbooks in Operations Research and Management Science: Network Models. North-Holland, 1995.

[10] C.-C. Jane and Y.-W. Laih, "Computing multi-state two-terminal reliability through critical arc states that interrupt demand," IEEE Transactions on Reliability, vol. 59, no. 2, pp. 338-345, 2010.

[11] Y. Niu, Z. Gao, and H. Sun, “An improved algorithm for solving all d-mps in multi-state networks,” Journal of Systems Science and Systems Engineering, vol. 26, no. 6, pp. 711-731, 2017.

[12] Z. Hao, W.-C. Yeh, and C.-F. Hu, "A novel multistate minimal cut vectors problem and its algorithm,” IEEE Transactions on Reliability, vol. 68, no. 1, pp. 291-301, 2018.

[13] M. Forghani-elahabad, N. Kagan, and N. Mahdavi-Amiri, “An mpbased approximation algorithm on reliability evaluation of multistate flow networks,” Reliability Engineering \& System Safety, vol. 191, p. 106566, 2019.

[14] W.-C. Yeh, "Fast algorithm for searching d-mps for all possible d,” IEEE Transactions on Reliability, vol. 67, no. 1, pp. 308315, 2018.

[15] M. Forghani-elahabad and L. H. Bonani, “Finding all the lower boundary points in a multistate two-terminal network,” IEEE Transactions on Reliability, vol. 66, no. 3, pp. 677-688, 2017.

[16] M. Forghani-elahabad and N. Kagan, "Reliability evaluation of a stochastic-flow network in terms of minimal paths with budget constraint,” IISE Transactions, vol. 51, no. 5, pp. 547-558, 2019.

[17] Y.-F. Niu, Z.-Y. Gao, and W. H. Lam, "Evaluating the reliability of a stochastic distribution network in terms of minimal cuts," Transportation Research Part E: Logistics and Transportation Review, vol. 100, pp. 75 - 97, 2017.

[18] M. Forghanielahabad and N. Mahdavi-Amiri, “A new efficient approach to search for all multi-state minimal cuts,” IEEE Transactions on Reliability, vol. 63, no. 1, pp. 154-166, 2014.

[19] L. Ling, X.-b. Xiao, J.-y. Xiong, L. Zhou, Z.-f. Wen, and X.-s. Jin, “A 3 d model for coupling dynamics analysis of high-speed train/track system,” Journal of Zhejiang University Science A, vol. 15, no. 12, pp. 964-983 , 2014.

[20] I. Okamoto, “How bogies work,” Japan Railway \& Transport Review, vol. 18, pp. 52-61, 1998.

[21] Y.-K. Lin and C.-T. Yeh, "Maximal network reliability for a stochastic power transmission network,” Reliability Engineering \& System Safety, vol. 96, no. 10, pp. 1332-1339, 2011.

[22] A.-L. Barabasi,^ H. Jeong, Z. Neda,' E. Ravasz, A. Schubert, and T. Vicsek, "Evolution of the social network of scientific collaborations,” Physica A: Statistical mechanics and its applications, vol. 311, no. 3-4 , pp. 590-614, 2002.

[23] S. Rai and S. Soh, "A computer approach for reliability evaluation of telecommunication networks with heterogeneous linkcapacities,” IEEE Transactions on reliability, vol. 40, no. 4, pp. 441-451, 1991.

[24] X. Dong, "How high-speed emus works and their structure characters," 2007.

[25] L. Kou, Y. Qin, L. Jia, and Y. Fu, "Multistate reliability evaluation of bogie on high speed railway vehicle based on the network flow theory,” International Journal of Software Engineering and Knowledge Engineering, vol. 28, no. 04, pp. 431-451, 2018.

[26] D. Fulkerson and L. Ford, Flows in networks. Princeton University Press, 1962. 
[27] R. Mishra and S. K. Chaturvedi, “A cutsets-based unified framework to evaluate network reliability measures,” IEEE Transactions on reliability, vol. 58, no. 4, pp. 658-666, 2009.

\section{Authors' Profiles}

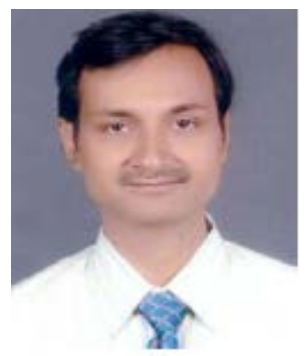

Rajesh Mishra is currently working as an assistant professor in School of Information and Communication Technology, Gautam Buddha University Greater Noida, Delhi NCR (India). He received his B.E. (Electronics Eng.), M.Tech and Ph. D. degree (Reliability Eng.) from Reliability Engineering Centre, IIT Kharagpur (India) in year 2000, 2004, and 2009, respectively. He has research interest in the area of reliability engineering, layout design for capacitated networks, and network optimization. He has published papers in several international journals such as IJPE, IEEE, RESS, and QTQM.

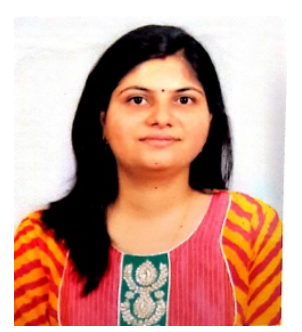

Vartika Sharma has received B.E. and M.Tech degree in Computer Science and Engineering from Rajasthan University, Jaipur and Gautam Buddh Technical University, Lucknow, respectively with Honours. Currently, she is working towards her doctorate degree from Gautam Buddha University, Greater Noida, India. She is a member of professional societies like CSI, ISTE, and IAENG etc. Her recent research activities include reliability analysis, stochastic flow networks, optimization and modelling.

How to cite this paper: Vartika Sharma, Rajesh Mishra. " Reliability Evaluation of High Speed Train Bogie System Based on Stochastic Network Flow Model ", International Journal of Mathematical Sciences and Computing (IJMSC), Vol.6, No.5, pp.29-36, 2020. DOI: 10.5815/IJMSC.2020.05.03 